\title{
Práticas de letramentos com escrita científica em artigos de impacto na área de ciências da vida e biomedicina
}

\author{
Mariana Aparecida Vicentini* \\ Adriana Fischer ${ }^{* *}$
}

\begin{abstract}
Resumo
$\mathrm{Na}$ atual conjuntura de avaliação da educação superior, a autocitação, concebida como um movimento em que o pesquisador cita seu trabalho anterior em um trabalho em desenvolvimento, é uma prática que tem ganhado destaque em distintas áreas de conhecimento, com ênfase à Área de Ciências da Vida e Biomedicina. Este artigo tem por objetivo discutir usos da autocitação em artigos de alto impacto da referida área, publicados no periódico Nature, nos anos de 2017 e 2018. O enfoque aos cinco artigos selecionados, na base Web of Science (WoS), é qualitativo, essencialmente sob a perspectiva sociocultutal dos estudos dos letramentos, que compreendem a escrita científica como prática social, condicionada ao contexto sóciohistórico de produção, ideologias e perpassada por relações de poder. Os resultados parecem indicar um padrão da grande área, com altos índices de autocitação distribuídos entre as seções do artigo, que parecem representar movimentos característicos da escrita científica, como contribuição para a área, reivindicação de crédito pelo trabalho do pesquisador, indicar interação com pesquisas anteriores, mais do que uma prática que contribuiria para a elevação de índices bibliométricos dos pesquisadores.

Palavras-chave: Escrita científica. Autocitação. Práticas de Letramentos. Ciências da Vida e Biomedicina. Nature.
\end{abstract}

\footnotetext{
* Universidade Regional de Blumenau (FURB). Mestre em Educação (FURB). Doutoranda em Educação (FURB). Bolsista de demanda social Capes. ORCID: https://orcid.org/0000-0001-6256-2904.

** Universidade Regional de Blumenau (FURB), Doutora em Linguística (UFSC). Docente no Centro de Ciências da Educação, Artes e Letras e no Programa de Pós-Graduação em Educação (FURB). ORCID: https://orcid.org/0000-0001-9787-2814.
}

Cadernos CESPUC de Pesquisa. Série Ensaios. n.39, $2^{\circ}$ Sem./2021, p. 36-51. e-ISSN: 2358-3231 (OJS). Recebido em: 10/11/2021. Aceito em: 15/12/2021. 


\title{
Literacy practices with scientific writing in impact articles in the field of life sciences and biomedicine
}

\author{
Mariana Aparecida Vicentini \\ Adriana Fischer
}

\begin{abstract}
In the current context of higher education assessment, self-citation, conceived as a movement in which researchers cite their previous work in a work in progress, is a practice that has gained prominence in different areas of knowledge, with emphasis on the field of Life Sciences and Biomedicine. This article, therefore, aims to discuss uses of self-citation in high-impact articles in that field, published in the journal Nature, in 2017 and 2018. The focus on the five selected articles, from the Web of Science database, is qualitative, essentially under the sociocultural perspective of the Literacy Studies, which understand scientific writing as a social practice, conditioned to the socio-historical context of production, ideologies and permeated by power relations. The results seem to indicate a pattern of the field, with high self-citation rates distributed among the sections of the article. This pattern seems to represent characteristic shifts of scientific writing, such as contribution to the field, claiming credit for the researcher's work, indicating interaction with previous research, more than a practice that would contribute to raising the bibliometric indexes of researchers.
\end{abstract}

Keywords: Scientific writing. Self-citation. Literacy Practices. Life Sciences and Biomedicine. Nature.

Cadernos CESPUC de Pesquisa. Série Ensaios. n.39, $2^{\circ}$ Sem./2021, p. 36-51. e-ISSN: 2358-3231 (OJS). Recebido em: 10/11/2021. Aceito em: 15/12/2021. 


\section{Introdução}

As discussões aqui estabelecidas representam um recorte de uma pesquisa longitudinal (2020-2023), que toma como objeto práticas de letramentos com escrita científica, em diferentes áreas de conhecimento, dentre as quais a de Ciências da Vida e Biomedicina. ${ }^{1}$ Para este artigo, nos propomos a discutir usos da autocitação em artigos de alto impacto desta grande área, publicados na revista Nature, nos anos de 2017 e 2018.

A justificativa para o desenvolvimento deste estudo se ampara no fato de que, no percurso de escrita científica, se revela, de maneira expressiva, um modelo de atividade científica fortemente balizado pelos traços das ciências experimentais e, ainda, impera ou se denuncia, fortemente, um modelo de racionalidade científica construído pelo domínio das ciências naturais, tal como descrito por Santos (2009, p. 21) como "um modelo totalitário, na medida em que nega o caráter racional a todas as formas de conhecimento que não se pautam pelos seus princípios epistemológicos". Esta perspectiva universalizante da ciência, para Santos (2009), atinge a escrita científica, que ainda é permeada por tabus linguísticos. Schwarze (2008, p. 4) exemplifica tal afirmação citando que, na escrita científica, a noção de estilo pode ser compreendida como "um sistema de características a respeitar de modo a apresentar a ciência por meio da linguagem”, sendo o estilo de um trabalho científico avaliado com base na aplicação de um sistema de regras gerais para a escrita científica, cuja transgressão levaria a uma perda de conhecimentos.

Somado a esses fatores que, na maioria das vezes, acabam por dar maior visibilidade a trabalhos produzidos por áreas ditas de maior status, e fazendo a comunidade científica contestar o que verdadeiramente conta como ciência, há o fato de que a Coordenação de Aperfeiçoamento de Pessoal de Nível Superior (CAPES), em reuniões com coordenadores de programas de pós-graduação, tem debatido a possibilidade de extinção do qualis ${ }^{2}$ e sua substituição por um modelo de avaliação que considere inovação, impacto e inserção social como grandes critérios de avaliação da área de educação e outras todas também. De acordo com a Capes,

\footnotetext{
1 Classificação adotada pela Web of Science para se referir à área de Ciências Biológicas.

2 O Qualis Capes é um sistema que faz a classificação da produção científica dos programas de pós-graduação brasileiros. 
O Qualis cumpriu um papel importante para a qualificação da produção da pósgraduação. Dados os novos recursos e funcionalidades nos instrumentos de TI e consolidação de banco de dados surgidos no tempo entre a última avaliação [...], a avaliação da produção, em cada área de avaliação, deve se dar a partir de métricas internacionais estabelecidas e amplamente aceitas na comunidade. Neste sentido, a Comissão propõe a extinção do Qualis a partir do próximo ciclo avaliativo (2021-2024) (CAPES, 2019, p. 25, grifos nossos).

No ano de 2018, a WoS ${ }^{3}$ divulgou o Clarivate Report (2018), um relatório encomendado pela Capes, que apresenta uma visão geral da pesquisa no Brasil, dos últimos seis anos. O impacto de uma publicação, na WoS, é medido pelo número de vezes que o artigo indexado na base foi citado, sem considerar quaisquer especificidades de cada área ou de seus modos de escrita. A partir desses novos meios de avaliação, uma das facetas da citação que vem tomando grandes proporções em diferentes áreas do conhecimento é o uso da autocitação em trabalhos científicos. De forma bastante sucinta, a autocitação é definida, neste estudo, como um movimento em que "o autor cita seu trabalho anterior em trabalhos acadêmicos subsequentes" (IOANNIDIS, 2015, p. 7, tradução nossa). ${ }^{4}$

Com vistas a discutir os usos da autocitação no atual contexto de avaliação da pesquisa e a responder ao objetivo proposto, apresentamos uma pesquisa qualitativa (BOGDAN; BIKLEN, 1994), com discussões orientadas pelos Estudos dos Letramentos (BARTON; HAMILTON, 2000; STREET, 2003; GEE, 2001), com apoio dos estudos sobre escrita científica desenvolvidos por Hyland $(2003 ; 2007)$, que nos permitem pensar os usos da autocitação na área de Ciências da Vida e Biomedicina, a partir de um olhar que compreende esta prática como integrada a aspectos sociais mais amplos, de interação e integração em diversos contextos e discursos.

Além dos diálogos desta introdução, este artigo ainda apresenta uma seção em que são discutidos enfoques teóricos que embasam este estudo; uma seção destinada à delimitação das principais escolhas metodológicas que orientaram a investigação; a seção de análises, em que são discutidos os usos da autocitação em artigos da área de Ciências da Vida e Biomedicina à luz da teoria proposta e, por fim, apresentamos as principais considerações acerca deste trabalho.

3 A plataforma Web Of Science será descrita na seção metodológica deste artigo.

4 The author cites his/her previous work in subsequent scholarly works. 


\section{A escrita científica como prática social}

Lançar um olhar à escrita científica em distintas áreas do conhecimento, neste caso, à biologia, pela perspectiva dos estudos dos letramentos, nos permite desvelar elementos norteadores à processos de avaliação que, por vezes, conforme mencionado na seção anterior, acabam por favorecer áreas ditas de maior status em detrimento de outras, sem considerar os processos subjetivos de produção de cada campo de saber. Ainda, tal enfoque nos permite examinar os textos como práticas disciplinares, que se estabelecem em um movimento que oscila entre o individual e o coletivo, dos limites da página para as atividades dos seres sociais (HYLAND, 2007).

Para esta discussão, portanto, cabe retomar a compreensão de que os estudos dos letramentos compreendem a linguagem escrita como uma prática flexível, de cunho social e, portanto, ideológicas, perpassadas por relações de poder e sócio-historicamente situadas. Dessa vertente, o conceito principal que sustenta nossas discussões é o de prática de letramentos, ou seja, as formas culturais que as pessoas utilizam a linguagem, oral ou escrita, em suas vidas (BARTON; HAMILTON, 2000). As práticas de letramentos não são observáveis, pois, além da leitura, escrita e oralidade, nelas estão envolvidos valores, atitudes, sentimentos, relacionamentos pessoais, relações de poder, discursos e modos como as pessoas utilizam e dão sentido aos letramentos. Além de relacionadas a questões culturais e sociais, as práticas estão relacionadas diretamente a

Discursos, com D maiúsculo (GEE, 2001), que são recorrentes em diferentes campos e envolvem, além da linguagem, [...] modos de falar, ouvir, escrever, ler, agir, interagir, acreditar, valorizar, sentir e usar vários objetos, símbolos, imagens, ferramentas e tecnologias, com a finalidade de ativar identidades e atividades significativas, socialmente situadas (GEE, 2001, p. 719, tradução nossa). ${ }^{5}$

Segundo Gee (2001), a principal característica dos Discursos é a de que são ideológicos, pois envolvem um conjunto de valores, pontos de

\footnotetext{
5 A Discourse with a capital " $\mathrm{D}$ " is composed of distinctive ways of speaking/listening and often, too, writing/reading coupled with distinctive ways of acting, interacting, valuing, feeling, dressing, thinking, believing, with other people and with various objects, tools, and technologies, so as to enact specific socially recognizable identities engaged in specific socially recognizable activities.
} 
vista sobre a relação entre as pessoas, sobre a distribuição de bens sociais e indicam quem são os insiders em determinadas práticas de letramentos. Discursos, nesse sentido, são nomeados por Gee (2001) como kits de identidades, pois possibilitam explicar as múltiplas identidades sociais de um sujeito e fornecer pistas sobre seus modos de inserção em práticas de letramentos de um determinado campo.

As práticas, portanto, além de se tratarem de um processo subjetivo, são processos sociais por meio dos quais pessoas se conectam umas com as outras e incluem o compartilhamento de cognições representadas em ideologias e identidades sociais. O conceito de práticas é relevante, em nossas discussões, pois abarca padrões que tenham a ver com o uso da autocitação na grande área em enfoque, tratando de associá-lo a algo mais amplo, de uma natureza cultural e social (STREET, 2003).

Nessa perspectiva de concepção da escrita científica, amparadas por Curry e Lillis (2016), compreenderemos os sujeitos que escrevem não como indivíduos isolados, mas como pesquisadores inseridos em um contexto social específico, convivendo com relações de poder no campo em análise. Da mesma forma, tomando por base artigos de impacto publicados na referida área, a autocitação, pela perspectiva em enfoque, é discutida não apenas como um elemento discursivo integrante do texto científico, mas como uma prática atrelada a um contexto de produção específico, perpassado por distintas identidades, relações de poder e ideologias do próprio campo e dos sujeitos nele inseridos.

$\mathrm{Na}$ seção seguinte, apresentamos as principais decisões metodológicas que orientaram o desenvolvimento deste artigo.

\section{Escolhas metodológicas}

A partir das discussões estabelecidas na seção introdutória, acerca de novas formas de avaliação da produção científica em distintas áreas de conhecimento divulgadas pela Capes, as análises se voltam a artigos indexados na Base de dados WoS. A escolha por esta base se dá, pois, em 2019, a WoS divulgou o Clarivate Report (2018), um relatório encomendado pela Capes, que apresenta uma visão geral da pesquisa no Brasil, dos últimos 
seis anos. É com base no referido relatório que a grande área selecionada para esta investigação é a de Ciências da Vida e Biomedicina.

A justificativa pela escolha dessa área se dá, principalmente, pelos dados fornecidos por este relatório (2018), dentre os quais: a) a maioria dos trabalhos de pesquisadores brasileiros são publicados nas áreas de Ciências da Vida; b) um terço dos trabalhos da área de Ciências da Vida são elaborados a partir de parcerias com pesquisadores internacionais; c) é a área em que o número de citações se acumula mais rapidamente; d) as áreas mais produtivas de pesquisa para uma universidade são as áreas com maior produção em todo o Brasil, dentre as quais estão as Ciências da Vida e; e) existem focos de excelência, em termos de impacto de citações, na pesquisa brasileira, na área de Ciências da Vida.

Para esta investigação, serão analisados os cinco artigos mais citados da Revista Nature, indexados na WoS, no período de 2017 e 2018. Destacamos que a escolha da Nature se deu por ser o periódico com maior fator impacto no mundo, com um total de 42.779 (WoS, 2018). Posteriormente, foram elencados os artigos com maior índice de citação desse periódico, nos anos de 2017 e 2018:

\section{Quadro 1 - classificação dos cinco artigos mais citados no periódico}

Nature nos anos de 2017-2018, na Web of Science.

\begin{tabular}{|l|l|l|c|c|}
\hline Artigo & \multicolumn{1}{|c|}{ Título } & \multicolumn{1}{c|}{ Autores } & Ano & $\begin{array}{c}\text { Citações ao } \\
\text { artigo } \\
(2017-2018)\end{array}$ \\
\hline A1 & $\begin{array}{l}\text { Dermatologist-level } \\
\text { classification of skin cancer } \\
\text { with deep neural networks }\end{array}$ & $\begin{array}{l}\text { Andre Esteva, Brett } \\
\text { Kuprel, Roberto A. } \\
\text { Novoa, Justin Ko et } \\
\text { al. }\end{array}$ & 2017 & 2.630 \\
\hline A2 & $\begin{array}{l}\text { Unconventional } \\
\text { superconductivity in magic- } \\
\text { angle graphene superlattices }\end{array}$ & $\begin{array}{l}\text { Yuan Cao, Valla } \\
\text { Fatemi, Shiang Fang, } \\
\text { Kenji Watanabe, et al. }\end{array}$ & 2018 & 1.432 \\
\hline A3 & $\begin{array}{l}\text { Neurotoxic reactive } \\
\text { astrocytes are induced by } \\
\text { activated microglia }\end{array}$ & $\begin{array}{l}\text { Shane A. Liddelow, } \\
\text { Kevin A. Guttenplan } \\
\text { Laura E. Clarke, } \\
\text { Frederick C. Bennett, } \\
\text { et al. }\end{array}$ & 2017 & 1.519 \\
\hline
\end{tabular}




\begin{tabular}{|l|l|l|c|c|}
\hline A4 & $\begin{array}{l}\text { Mastering the game of Go } \\
\text { without human knowledge }\end{array}$ & $\begin{array}{l}\text { David Silver, Julian } \\
\text { Schrittwieser, Karen } \\
\text { Simonyan, Ioannis } \\
\text { Antonoglou, et al. }\end{array}$ & 2017 & 1.445 \\
\hline A5 & $\begin{array}{l}\text { Layer-dependent } \\
\text { ferromagnetism in a van der } \\
\text { Waals crystal down to the } \\
\text { monolayer limit }\end{array}$ & $\begin{array}{l}\text { Bevin Huang, } \\
\text { Genevieve Clark, } \\
\text { Efrén Navarro- } \\
\text { Moratalla, Dahlia R. } \\
\text { Klein, et al. }\end{array}$ & 2017 & 1.211 \\
\hline
\end{tabular}

Fonte: elaborado pelas pesquisadoras com base em Fischer, Grimes, Kosloski e Vicentini em 2021.

Esta seleção, em consequência, oportuniza análises de dados em torno da autocitação nos artigos, apresentadas na seção a seguir.

\section{Autocitação em artigos de ciências da vida e biomedicina}

A escrita científica é concebida, nesta investigação, como uma prática de letramento. Tal percepção permite lançar às publicações do campo científico em enfoque que contribui na compreensão das dinâmicas de visibilidade que artigos recebem em periódicos da área.

Conforme constatado em estudo realizado por Fischer, Grimes, Kosloski e Vicentini (2021), um aspecto que merece atenção na escrita científica da Nature é a utilização, dentro do periódico, das normas Vancouver, tipicamente utilizadas por periódicos das ditas Hard Sciences. 


\section{Unconventional superconductivity in magic-angle graphene superlattices}

Yuan $\mathrm{CaO}^{1}$, Valla Fatemi ${ }^{1}$, Shiang Fang ${ }^{2}$, Kenji Watanabe ${ }^{3}$, Takashi Taniguchi ${ }^{3}$, Efthimios Kaxiras $^{2,4} \&$ Pablo Jarillo-Herrero ${ }^{1}$

Strong interactions among particles lead to fascinating states of matter, such as quark-gluon plasmas, various forms of nuclear matter within neutron stars, strange metals and fractional quantum Hall states ${ }^{1-3}$ An intriguing class of strongly correlated materials is the unconventional superconductors, which includes materials with a range of superconducting critical temperatures $T_{c}$, from heavy-fermion and organic superconductors with relatively low $T_{\mathcal{c}}$ (a few to a few tens of kelvin) to iron pnictides and cuprates that can have $T_{c}>100 \mathrm{~K}$ (refs 4-8). Despite extensive experimental efforts to characterize these materials, unconventional superconductors are challenging to study theoretically because the models that are typically used to describe them cannot be solved exactly, motivating the development of alternative approaches for investigating and modelling strongly correlated systems. One approach is to simulate quantum materials with ultracold atoms trapped in optical lattices, although technical advances are necessary to realize $d$-wave superfluidity with ultracold atoms at lower temperatures than are currently possible $e^{9,10}$

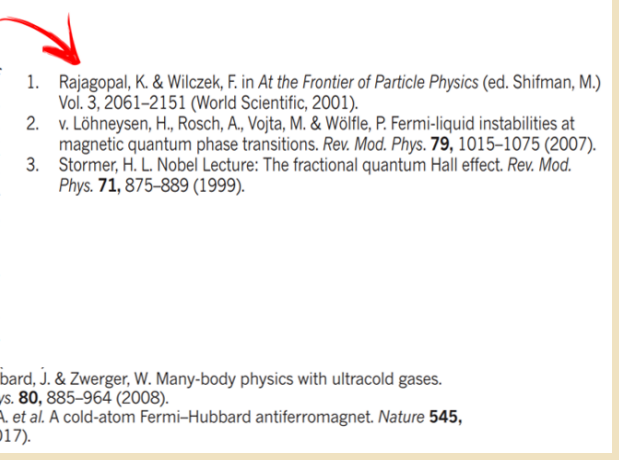

Fonte: dados da pesquisa em 2021.

De acordo com Rodrigues (2015), a escrita científica é orientada pelas normas do gênero, pelas normas das instituições em que o texto circula e pelas normas de formatação e publicação adotadas pelo periódico. Quando utilizadas, as referidas normas produzem sentidos, construídos sob certas condições que abarcam:

a) um locutor (aquele que diz, tendo em vista seus papéis sociais e comunicativos); b) um alocutário (aquele para quem se diz o que se tem a dizer, tendo em vista seus papéis sociais e comunicativos); c) um referente (o que dizer); d) uma forma de dizer (as estratégias para se dizer); e) um contexto em sentido estrito e em sentido lato (RODRIGUES, 2015, p. 359).

Nessa direção, relações de poder necessariamente parecem regular o que conta como conhecimento (STREET; LEA; LILLIS, 2015) e o modo como ele é materializado por meio da linguagem neste periódico e/ou campo científico, levando em conta os elementos mencionados por Rodrigues (2015). A relação entre as normas de publicação e o uso da autocitação, em nossas discussões, se aproxima, pois, as normas em enfoque parecem promover o apagamento de marcas que remetem à palavra alheia no corpo

Práticas de letramentos com escrita científica em artigos de impacto na área de ciências da vida e biomedicina 
do texto, diferentemente do que ocorre em textos escritos com base em outras normas, a exemplo de APA ou ABNT, que parecem permitir maior visibilidade dos autores no decorrer do texto do artigo.

Além disso, as formas e a extensão com que os pesquisadores se referem a si próprios e ao seu trabalho oferecem importantes insights sobre os pressupostos que eles sustentam sobre seu papel no processo de pesquisa e sobre as preferências epistemológicas e sociais de suas disciplinas (HYLAND, 2003). Nesse sentido, o Quadro 2, a seguir, apresenta a organização dos dados dos artigos analisados, com ênfase ao número de vezes que foram citados por outros autores, número de autores, referências e autocitações por referências. Cabe ressaltar que, nos artigos analisados, cada autocitação foi contabilizada apenas uma vez, independentemente do número de ocorrências no texto.

Quadro 2 - número de vezes que o artigo foi citado por outros autores, número de autores, referências e autocitações por referências.

\begin{tabular}{|c|c|c|c|c|}
\hline Artigo & Citado & $\begin{array}{c}\text { Número de } \\
\text { autores }\end{array}$ & $\begin{array}{c}\text { Número de } \\
\text { referências }\end{array}$ & $\begin{array}{c}\text { Número de autocitação } \\
\text { por referência }\end{array}$ \\
\hline A1 & 2.630 & 7 & 30 & 0 \\
\hline A2 & 1.432 & 7 & 65 & $13(20 \%)$ \\
\hline A3 & 1.519 & 21 & 48 & $16(33,33 \%)$ \\
\hline A4 & 1.445 & 17 & 69 & $11(15,94 \%)$ \\
\hline A5 & 1.211 & 14 & 37 & $5(13,5 \%)$ \\
\hline
\end{tabular}

Fonte: elaborado pelas pesquisadoras com base em Fischer, Grimes, Kosloski e Vicentini em 2021.

O uso da autocitação, segundo Hyland (2003), tem se mostrado uma estratégia (CANAGARAJAH, 2014) para pesquisadores atingirem a credibilidade no campo científico, considerando os modos de avaliação baseados em métricas internacionais. Nesse sentido, a partir do quadro 2, o uso da autocitação parece dar indícios de que grande parte dos pesquisadores estão se inserindo em uma prática dita dominante (STREET, 2003; BARTON; HAMILTON, 2000), presente e disseminada em um contexto socialmente institucionalizado que, por consequência, trará contribuições para a sustentação e sobrevivência deste campo específico. 
Uma publicação bem-sucedida, por exemplo, pode ajudar um pesquisador a obter crédito, que pode ser convertido em uma bolsa de pesquisa para financiar equipamentos e recrutar colegas, o que, por sua vez, gera mais dados que podem ser convertidos em argumentos, novas publicações e assim por diante (HYLAND, 2003).

Conforme mostra o Quadro 2, com exceção de A1, os artigos analisados apresentaram um alto índice de autocitações por referência, sendo A 2 com 13/65 (20\%), A3 com 16/48 (33\%), A 4 com 11/69 (16\%) e A5 com $5 / 37$ (13\%). Este padrão confirma as reflexões realizadas por Hyland (2003), sobre artigos da grande área de Ciências da Vida e Biomedicina, que possuem um número elevado de autocitações, um padrão da própria área.

É interessante destacar, segundo Silva (2018), que as diferentes posições que integram um campo apresentam distintos níveis de poder, o que resulta em uma constante luta para a definição de quem irá dominar determinado campo. No caso desta investigação, pode-se dizer que, de um lado estão os pesquisadores menos experientes, que não compreendem os modos de inserção em práticas de letramentos por meio do discurso científico e, de outro, pesquisadores experientes que compreendem a autocitação como uma prática de letramento científico que pode aumentar, ainda mais, seu status em um campo específico. Isso, pois, a autocitação, de acordo com Hyland (2003), parece se evidenciar como uma consequência retórica deste crescimento da competitividade, afetando a visibilidade de um autor entre colegas e o perfil nos índices de citação dos quais sua carreira pode depender. Além disso, ao situar-se na literatura que citam, os escritores aumentam sua autoridade, o que contribui na constituição uma identidade disciplinar sólida e no aumento da probabilidade de que seu trabalho seja aceito.

Com base nessas discussões, há indícios de que, apesar de A1 ser o artigo mais citado por outros autores, não há a presença de autocitações no trabalho e, ao contrário da discussão no parágrafo anterior, talvez a discussão em enfoque já seja legitimada dentro da área de conhecimento, não havendo a necessidade de adoção da autocitação como uma estratégia, diferentemente dos demais autores dos artigos em análise.

Ao investigar o perfil dos autores de A1 na plataforma Google Acadêmico, há indícios de que o grupo ainda não havia desenvolvido estudos acerca da temática do artigo em análise e esse pode ser um dos 
motivos pelo qual o grupo não tenha se autocidado no corpo do texto. Tal fato corrobora as discussões de Hyland (2003) e Canagarajah (2014), quando afirmam que a autocitação tem se mostrado uma importante ferramenta para pesquisadores atingirem a credibilidade no campo científico (HYLAND, 2003), com vistas a indicar contribuição para a área; reivindicar crédito por seu trabalho; sinalizar que estão fortemente por trás dele (CANAGARAJAH, 2014). A dependência de argumentos em redes de referências não apenas sugere uma progressão cumulativa e linear de conhecimento, mas atua para localizar os escritores e suas reivindicações dentro de uma estrutura disciplinar reconhecida. Quando tal referência é para o próprio trabalho anterior dos autores, isso claramente fornece suporte adicional, enfatizando seu envolvimento sustentado em um esforço profissional comum (HYLAND, 2003). No caso de A1, como não havia trabalhos anteriores realizados pelo grupo, que pudessem apontar os elementos indicados por Hyland (2003) e Canagarajah (2014), não foi possível ou necessário, o uso de autocitação.

O Quadro 3, a seguir, apresenta o uso de autocitações de acordo com cada seção dos artigos analisados:

Quadro 3 - Autocitação por seção dos artigos

\begin{tabular}{|l|l|l|l|l|l|}
\hline & A1 & A2 & A3 & A4 & A5 \\
\hline Resumo & - & 0 & 0 & 0 & - \\
\hline Introdução & 0 & $3(17,6 \%)$ & $4(19 \%)$ & $4(23,5 \%)$ & $4(44,44 \%)$ \\
\hline Aporte Teórico & 0 & $3(17,6 \%)$ & - & $1(5,8 \%)$ & $2(22,22 \%)$ \\
\hline Resultados e Discussões & 0 & $2(11,7 \%)$ & $8(38 \%)$ & $3(17,64)$ & $1(11,11 \%)$ \\
\hline Conclusões & 0 & - & $2(9,5 \%)$ & 0 & 0 \\
\hline Métodos & 0 & $9(53 \%)$ & $7(33,33 \%)$ & $9(52,94 \%)$ & $2(22,22 \%)$ \\
\hline
\end{tabular}

Fonte: elaborado pelas pesquisadoras com base em Fischer, Grimes, Kosloski e Vicentini em 2021.

A área de Ciências da Vida e Biomedicina, conforme o Quadro 3, apresenta um padrão de distribuição de autocitação por seção do artigo, sendo concentrada na introdução, resultados e discussões e métodos. Uma possível justificativa para maiores índices de autocitação nessas seções pode 
estar atrelada ao fato de que é na introdução que o autor estabelece seu nicho de pesquisa e delimita demais pesquisas em torno do objeto em análise; na seção metodológica, o uso da autocitação pode aparecer como forma de o pesquisador indicar sua participação no percurso de sistematização dos dados e, finalmente, nos resultados e discussões, a autocitação talvez se manifeste pelo diálogo que o pesquisador estabelece com pesquisas em discussão ou com estudos anteriores. Nesse sentido, referido campo se configura como uma comunidade de prática (WENGER, 2004), em que a sistematização dos conhecimentos e a emergência de posicionamentos críticos vão acontecendo gradativamente (FISCHER, 2007), em virtude, principalmente, das necessidades de interação com o meio científico e com o contexto global (CANAGARAJAH, 2014) de avaliação da pesquisa.

Neste caso, compreendemos o uso da autocitação, nos artigos em análise, não apenas como uma imposição atual do campo e das novas formas de avaliação estabelecidas: i) pesquisadores avaliados não mais pelo qualis de suas publicações, mas pelo número de vezes em que são citados em grandes bases de dados como a WoS, ii) também, como uma prática disciplinar dentro da grande área de Ciências da Vida e Biomedicina, que possibilita aos pesquisadores legitimarem trabalhos desenvolvidos pelo grupo como um todo.

\section{Considerações finais}

A partir do objetivo de discutir usos da autocitação em artigos de alto impacto da área de Ciências da Vida e Biomedicina, publicados na revista Nature, nos anos de 2017 e 2018, foi possível interpretar, por meio das análises, que relações de poder parecem permear e regular as práticas de letramentos na escrita dos artigos analisados. Os dados parecem indicar que, assim como possivelmente ocorre em demais áreas de conhecimento, o campo de Ciências da Vida e Biomedicina é permeado por práticas institucionalizadas, orientadas por forças que levam os sujeitos a incorporarem determinados modos de ser, agir, se posicionar, refletidos na escrita, desvelando, portanto, um estilo científico de escrita da área. 
Entretanto, a partir das novas normas de avaliação implementadas, em que sujeitos serão avaliados pelo número de citações que recebem, a autocitação parece ser uma prática atual que vem se inserindo no meio científico, à qual pesquisadores precisam, rapidamente, aderir, para garantir a sobrevivência no/do campo. Destacamos que nossas discussões em torno da autocitação vão ao encontro da necessidade de um posicionamento em relação aos modos de avaliação da ciência, que não têm dado a devida atenção ao peso das instituições, ao jogo de influências entre áreas de conhecimento, disciplinas e mesmo aos percursos individuais de pesquisadores. Nesse sentido, ao invés de uma avaliação homogênea, no contexto de produção e disseminação do conhecimento deve-se considerar relações de força e de formas de desigualdade (entre áreas, disciplinas, línguas, instituições) da própria disciplina e seu peso na cultura científica.

\section{Referências}

BARTON, D.; HAMILTON, M. Literacy practices. BARTON, D.; HAMILTON M.; IVANIC, R. Situated literacies: reading and writing in context. London: Routledge, 2000. p. 7-15.

BOGDAN, R.; BIKLEN, S. A escolha de um estudo. In: BOGDAN, R.; BIKLEN, S. Investigação qualitativa em educação: uma introdução à teoria e aos métodos. Portugal: Porto, 1994, p. 85-88

CANAGARAJAH, S. Local Knowledge When Ranking Journals: Reproductive Effects and Resistant Possibilities. Education Policy Analysis Archives, v. 22, n. 28, p. 2-21, 2014. Disponível em: Acesso em:

CAPES - Coordenação de Aperfeiçoamento de Pessoal de Nível Superior. Proposta de Aprimoramento da Avaliação da Pós-Graduação Brasileira para o Quadriênio 2021-2024: Modelo Multidimensional, 2020. Disponível em: https://www.gov.br/capes/pt-br/centrais-de-conteudo/25052020relatorio-final-2019-comissao-pnpg-pdf. Acesso em: 12 jan. 2021.

CURRY, M. J.; LILLIS, T. Estratégias e táticas na produção do conhecimento acadêmico por pesquisadores multilíngues. [Traduzido por Raquel 
Salek Fiad e Flávia Danielle Sordi Silva Miranda]. In: FIAD, R. S. (org.). Letramentos acadêmicos: contextos, práticas e percepções. São Carlos: Pedro \& João editores, 2016.

FISCHER, A.; GRIMES, C.; KOSLOSKI, E. R.; VICENTINI, M. Padrões da autocitação em artigos de alto impacto da revista Nature. Revista IberoAmericana de Estudos em Educação, Araraquara, v. 16, n. 1, p. 276-291, jan./mar. 2021.

FISCHER, A. A construção de letramentos na esfera acadêmica. 2007. Tese (Doutorado em Linguística) - Programa de Pós-Graduação em Linguística, Universidade Federal de Santa Catarina, Florianópolis, 2007.

GEE, J. P. Reading as situated language: a sociocognitive perspective. Journal of adolescent \& adult literacy, v.8, n. 44, 2001. p. 714-725.

HYLAND, K. Disciplinary cultures, texts and interactions. In: HYLAND, K. Disciplinary discources: social interactions in academic writing. Michigan: Michigan classics ed., 2007. 228 p.

HYLAND, K. Self-citation and self-reference: credibility and promotion in academic publication. Journal of the American Society of Information, Science and Technology, n. 54, n. 3, p. 251-259, 2003.

IOANNIDIS, J. P. A. A generalized view of self-citation: Direct, co-author, collaborative, and coercive induced self-citation. Journal of Psychosomatic Research, n. 78, p. 7-11, 2015.

RODRIGUES, D. L. D. I. Para além da normalização: aspectos discursivos das normas de padronização. SCRIPTA, Belo Horizonte, v. 19, n. 36, p. 357-368, 2015

SANTOS, B. S. A Non-Occidentalist West?: Learned Ignorance and Ecology of Knowledge. Theory, Culture \& Society, v. 26, n. 7, p. 103-125, 2009.

SCHWARZE, S. Le style, c'est l'homme. Unité et pluralité du discours scientifique dans les langues romanes. Francfort-sur-le-Main, Lang, 2008. $344 \mathrm{p}$. 
SILVA, W. P. Da praxiologia bourdieusiana à sociologia psicológica de Lahire: estabelecendo um diálogo disposicionalista. Estudos de Sociologia, v. 2, n. 24, p. 37-61, 2018.

STREET, B.; LEA, M. R.; LILLIS, T. Revisiting the question of transformation in academic literacies: the ethnographic imperative. In: LILLIS, T.; HARRINGTON, K.; LEA, M. R.; MITCHELL, S. Working with academic literacies: case studies towards transformative practice. Anderson, South Carolina: Parlor Press; Fort Collins, Colorado: WAC Clearinghouse, 2015.

STREET, B. Abordagens Alternativas ao Letramento e Desenvolvimento. Apresentado durante a Teleconferência Unesco Brasil sobre 'Letramento e Diversidade'. Londres, 2003.

WEB OF SCIENCE GROUP. Research in Brazil: Funding excellence - Analysis prepared on behalf of CAPES by the Web of Science Group. Clarivate Analytics Company, 2018.

WENGER, E. Communities of practice: a brief introduction. 2004. Disponível em: https://wenger-trayner.com/introduction-to-communitiesof-practice/ Acesso em: 20 jan. 2021. 